\title{
Auxin is involved in arbuscular mycorrhizal fungi-promoted tomato growth and NADP- malic enzymes expression in continuous cropping substrates
}

Yu Wang ${ }^{1+}$, Wenze Zhang ${ }^{1+}$, Weikang Liu', Golam Jalal Ahammed ${ }^{2}$, Wenxu Wen', Shirong Guo ${ }^{1}$, Sheng Shu ${ }^{1 *}$ and $\operatorname{Jin} \operatorname{Sun}^{1 *}(1)$

\begin{abstract}
Background: Despite significant limitations of growth medium reuse, a large amount of organic substrate is reused in soilless cultivation of horticultural crops in China. Arbuscular mycorrhizal fungi (AMF) can promote nutrient absorption and improve plant tolerance to biotic and abiotic stresses. However, the mechanisms governing the effects of AMF on crop growth in organic continuous cropping substrates have not been elucidated.

Results: In this study, we showed that the inoculation of AMF in continuous cropping substrates promoted growth and root development, and increased the root and NADP-malic enzyme (NADP-ME) activity of tomato seedlings. Root transcriptome analysis demonstrated that the plant hormone signal transduction pathway was highly enriched, and 109 genes that positively correlated with the AMF-inoculated plant phenotype were obtained by gene set enrichment analysis (GSEA), which identified 9 genes related to indole acetic acid (IAA). Importantly, the levels of endogenous IAA in tomato seedlings significantly increased after AMF inoculation. Furthermore, the application of AMF significantly increased the expression levels of NADP-ME1 and NADP-ME2, as well as the activity of NADP-ME, and enhanced the root activity of tomato seedlings in comparison to that observed without inoculation of AMF. However, these effects were blocked in plants treated with 2,3,5-triiodobenzoic acid (TIBA), a polar transport inhibitor of IAA.
\end{abstract}

Conclusions: These results suggest that IAA mediates the AMF-promoted tomato growth and expression of NADP-MES in continuous cropping substrates. The study provides convincing evidence for the reuse of continuous cropping substrates by adding AMF as an amendment.

Keywords: Tomato, Arbuscular mycorrhizal fungi, Continuous cropping substrate, IAA, Root activity

\section{Background}

Soilless cultivation promotes crop growth and yield by providing the most suitable environment for root growth and development [1]. Due to the numerous advantages of organic substrate cultivation, such as improved crop

\footnotetext{
*Correspondence: shusheng@njau.edu.cn; sunj72@163.com

${ }^{+}$Yu Wang and Wenze Zhang contributed equally to this work.

${ }^{1}$ College of Horticulture, Nanjing Agricultural University, Nanjing 210095, China

Full list of author information is available at the end of the article
}

yield and quality, reduced pesticide, water and fertilizer use, simple operation and management, reduced investment cost for equipment, avoidance of soil continuous cropping obstacles, and suitability for green organic cultivation, this type of soilless cultivation has been widely practiced and currently accounts for over $75 \%$ of the total area of soilless cultivation in China [2]. The annual production and sales volume of organic substrates in China is approximately 10 million $\mathrm{m}^{3}$. At the same time,

(c) The Author(s). 2021 Open Access This article is licensed under a Creative Commons Attribution 4.0 International License, which permits use, sharing, adaptation, distribution and reproduction in any medium or format, as long as you give appropriate credit to the original author(s) and the source, provide a link to the Creative Commons licence, and indicate if changes were made. The images or other third party material in this article are included in the article's Creative Commons licence, unless indicated otherwise in a credit line to the material. If material is not included in the article's Creative Commons licence and your intended use is not permitted by statutory regulation or exceeds the permitted use, you will need to obtain permission directly from the copyright holder. To view a copy of this licence, visit http://creativecommons.org/licenses/by/4.0/ The Creative Commons Public Domain Dedication waiver (http://creativecommons.org/publicdomain/zero/1.0/) applies to the data made available in this article, unless otherwise stated in a credit line to the data. 
the same volume of the substrates used is produced every year. However, the physical and chemical properties of the used substrates deteriorate when reused, resulting in larger bulk density and lower $\mathrm{pH}$, which are not conducive to crop growth and development [3]. Studies have shown that the numbers of beneficial fungi decrease and those of harmful fungi increase in continuous cropping substrates [4-6]. In addition, the effective nutrient content, substrate enzyme activity, and root index in the rhizosphere environment significantly decrease with the increase in continuous cropping years [4, 5]. However, it is imperative to improve and reuse substrates to reduce the labor input and production costs. At present, few methods have been used to improve continuous cropping substrates, such as substrates disinfection and crop rotation $[4,6]$. Interestingly, the inoculation with beneficial microorganisms in the continuous cropping soil has been demonstrated to be an effective way to overcome continuous cropping obstacles [7]. However, the role of beneficial microorganisms in continuous cropping substrates is largely unknown.

Arbuscular mycorrhizal fungi (AMF) are ubiquitous soil microorganisms and obligate symbionts that are important components of terrestrial ecosystems and can form a mutually beneficial symbiosis with approximately $80 \%$ of vascular plant roots [8]. The glycoproteins produced by AMF hyphae can create a suitable rhizosphere environment and better conditions for the growth of almost all vascular plants [9]. AMF symbiosis not only increases the input of root carbon but also enhances carbon fixation in soil by reducing the decomposition of soil organic matter and the rhizosphere priming effect [10]. AMF endow soil and roots with a direct connection, enhance plant mineral nutrition, water acquisition and photosynthesis, and alleviate the adverse effects of abiotic stresses, such as heavy metals and drought stress [11-13]. Our previous study showed that AMF inoculation on seedling substrates can reduce the harm of saline-alkali land exerted on processing tomato [14]. In addition, the application of AMF can effectively overcome the continuous cropping obstacles in continuous cropping soil, to promote growth and development of many crops, such as pepper [15], soybean [16], and cucumber [17]. However, the associated mechanism of AMF-promoted growth in continuous cropping systems has not been elucidated.

In this study, we investigated the effect and mechanism of AMF on the cultivation of tomato in continuous cropping organic substrates. The results showed that the inoculation of AMF in the continuous cropping substrates promoted the growth and increased the activity of root and NADP-malic enzyme (NADP-ME), and the level of indole acetic acid (IAA) in tomato seedlings. However, these effects were compromised when plants were treated with IAA polar transport inhibitor, indicating that IAA played a critical role in AMF-promoted plant growth. The results provide a theoretical and practical basis for the cultivation of tomato in continuous cropping substrates.

\section{Results}

Effects of AMF on root morphology, plant growth and net photosynthetic rate in tomato seedlings

To test the role of AMF in continuous cropping substrates, we first compared the growth of tomato in continuous cropping substrates with or without inoculation of AMF. AMF successfully colonized tomato roots, and the colonization rate was approximately $32 \%$ in tomato seedlings cultivated in the continuous cropping substrate inoculation with AMF (AM) (Additional file 1: Figure S1). The inoculation of AMF significantly promoted the root growth compared with the tomato seedlings cultivated in the continuous cropping substrate (NM) (Fig. 1a). The root length, total root surface area, total root volume, average root diameter, and the number of root tips of AM seedlings increased by $32,30,23,6$, and $27 \%$, respectively, and the fresh weight and dry weight of roots increased by 92 and 54\%, respectively, compared with those of the NM seedlings (Additional file 2: Table S1). The plant height, stem diameter, fresh weight and dry weight of AM seedlings increased by $29,14,30$, and $23 \%$, respectively, at $40 \mathrm{~d}$ in comparison to those observed for the NM seedlings (Fig. 1b). Furthermore, the net photosynthetic rate $(\mathrm{Pn})$ and yield of AM seedlings were higher than those observed for the NM seedlings (Fig. 1c; Additional file 3: Figure S2). The results showed that the inoculation of AMF significantly promoted the growth of tomato seedlings in continuous cropping substrates.

\section{Effects of AMF on root activity and NADP-dehydrogenase activity}

AMF have been shown to improve root development in plants under adverse conditions [18, 19]. Furthermore, the activity of NADP-dehydrogenase is closely related to the root activity of plants [20]. Therefore, we measured the root activity of NM and AM seedlings at $40 \mathrm{~d}$ and the NADP-dehydrogenase activity of tomato roots at 20, $25,30,35$, and $40 \mathrm{~d}$ after treatment. The root activity of AM seedlings was $90 \%$ higher than that of NM seedlings (Fig. 2a). Furthermore, the NADP-ME activity of AM seedlings was significantly higher than that of NM seedlings (Fig. 2b). However, there was no significant difference in the activity of NADP-isocitrate dehydrogenase (NADP-IDCH), glucose-6-phosphate dehydrogenase (G6PDH), or 6-phosphate gluconate dehydrogenase (6PGDH), between NM and AM seedlings (Fig. 2c-e). 
$\mathbf{a}$

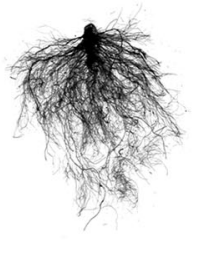

NM

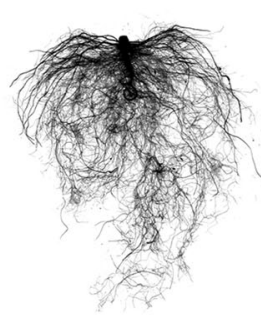

AM
C

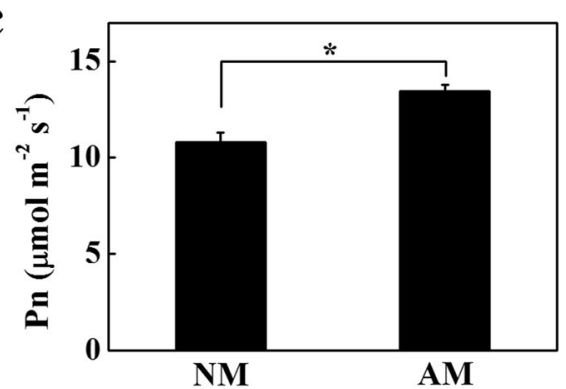

b
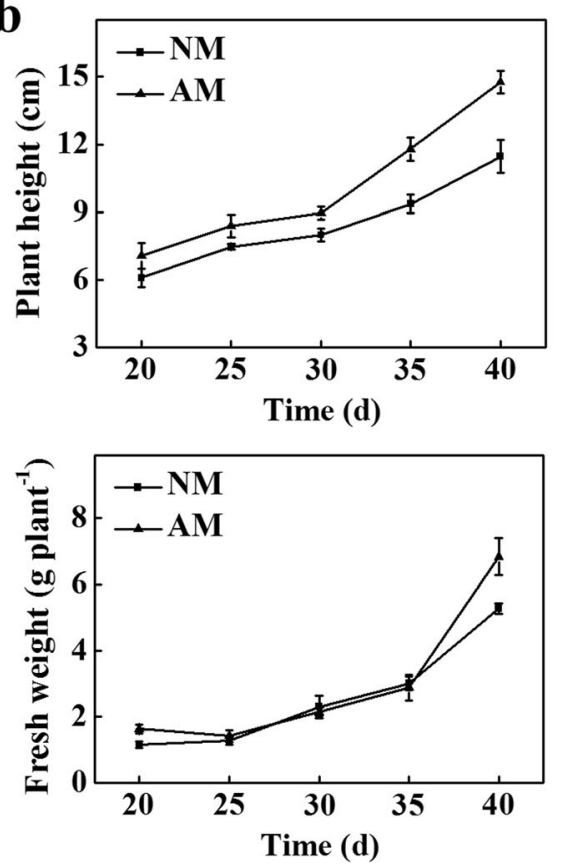
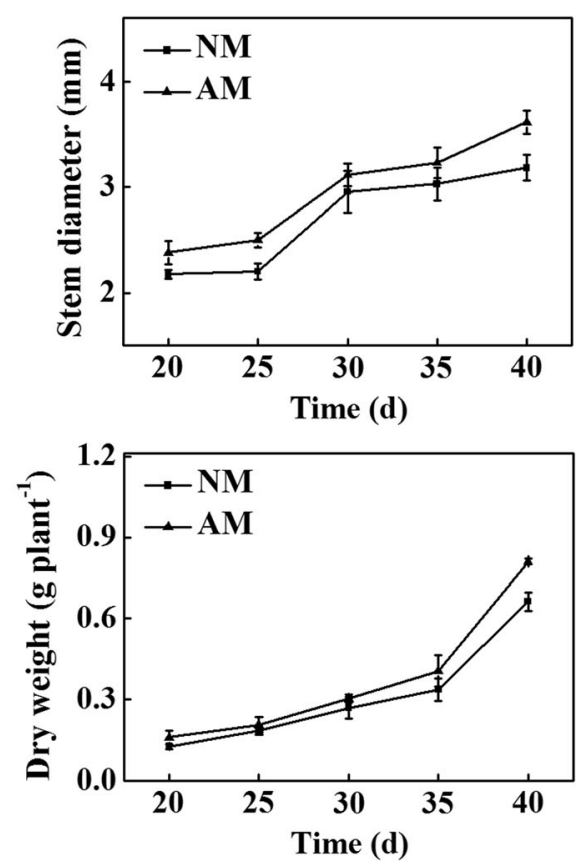

Fig. 1 Arbuscular mycorrhizal fungi (AMF) promoted growth of tomato seedlings in the continuous cropping substrates. a The root morphology of NM and AM seedlings at $40 \mathrm{~d}$. b The plant height, stem diameter, fresh and dry weight of NM and AM seedlings. $\mathbf{c}$ The net photosynthetic rate $(\mathrm{Pn})$ of $\mathrm{NM}$ and $\mathrm{AM}$ seedlings at $40 \mathrm{~d}$. The results represent the means $\pm \mathrm{SE}$. Three independent experiments were performed, with similar results. * represent significant difference. NM, tomato seedlings cultivated in continuous cropping substrate. AM, tomato seedlings cultivated in continuous cropping substrate inoculation with AMF

\section{Transcriptome analysis}

To further investigate how AMF increased root activity, we used transcriptome analysis to identify the differentially expressed genes (DEGs) in the roots between NM and AM seedlings. Illumina sequencing was performed on 6 root cDNA libraries (NM and AM treatments, each with 3 biological replicates) after cultivation of tomato seedlings for $30 \mathrm{~d}$. After removing the low-quality reads, we obtained 397,526,276 clean reads (Additional file 4: Table S2). The average values of Q20, Q30, and clean read rate of the three NM libraries were 98, 92, and $92 \%$, and these indexes of AM libraries were 98, 92, and 93\%, respectively (Additional file 4: Table S2). Over 92\% of the reads matched the tomato genome, and more than $90 \%$ of the reads were uniquely mapped (Additional file 5: Table S3). The clustering heat map intuitively showed the gene expression of NM and AM with relatively consistent and good repeatability (Additional file 6: Figure S3). Moreover, there were 4522 genes with significant differences between NM and AM, including 2566 downregulated genes and 1956 upregulated genes (Additional file 7: Table S4).

\section{Gene ontology (GO) enrichment analysis}

The DEGs of AM seedlings were significantly enriched for $24 \mathrm{GO}$ items in biological processes, 16 GO items in cellular components, and 11 GO items in molecular function (Fig. 3a; Additional file 8: Table S5). The GO enrichment analysis results for biological processes showed that GO terms, such as hydrogen peroxide decomposition process (GO:0042744), defense response (GO:0006952), response to oxidative stress (GO: 


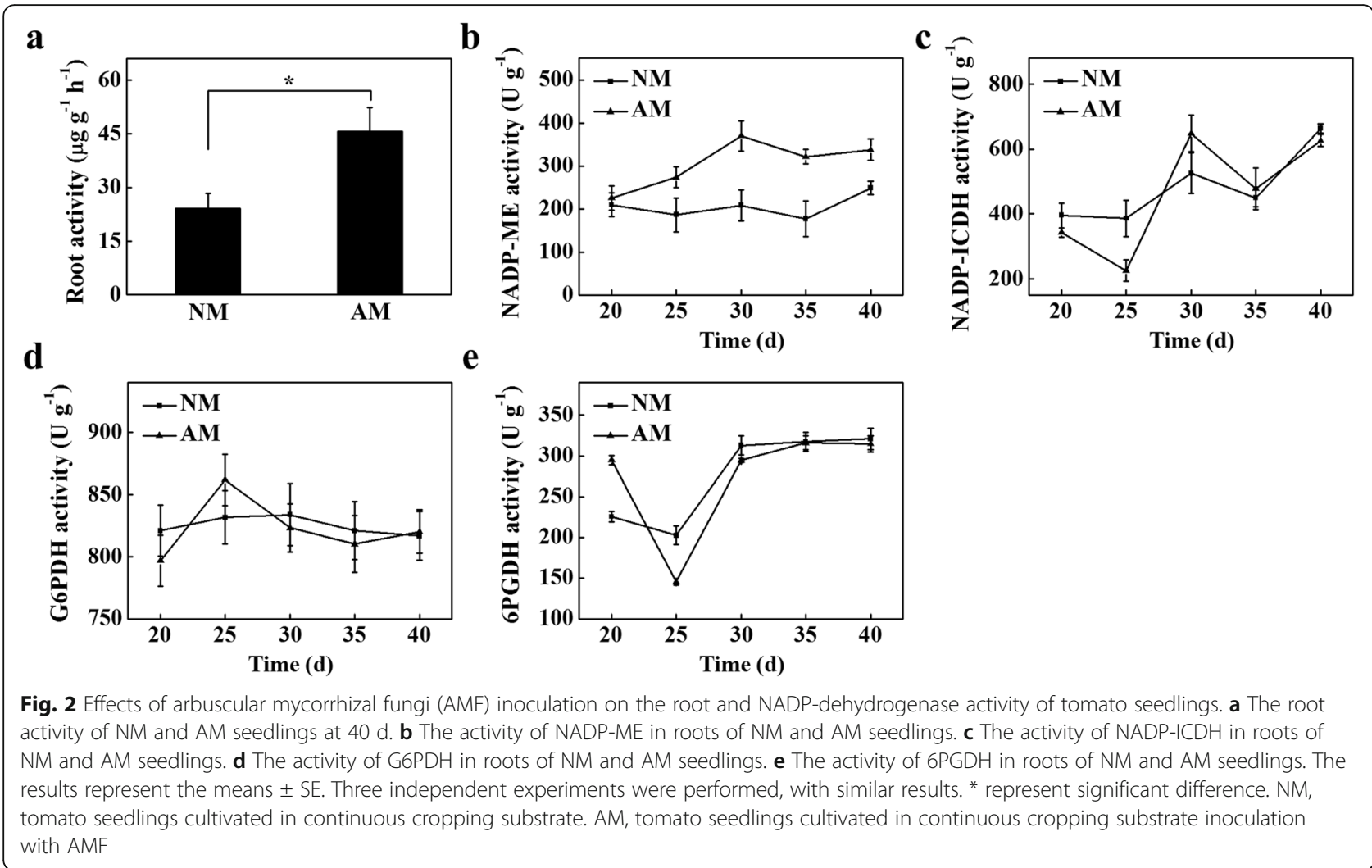

0006979), and response to biotic stimulus (GO:0009607) were enriched after AMF inoculation (Fig. 3b; Additional file 8: Table S5). The GO enrichment analysis results for cell components indicated that more significantly different genes were concentrated in the GO terms related to the cell membrane, such as extracellular region (GO: 0005576), integral component of membrane (GO: 0016021), plasma membrane (GO:0005886), and plant cell wall (GO:0009505) (Fig. 3c; Additional file 8: Table S5). The GO enrichment analysis results for molecular functions showed that GO items such as DNA-binding transcription factor activity (GO:0003700), peroxidase activity (GO:0004601), oxidoreductase activity (GO: 0016491), iron ion binding (GO:0005506), and monooxygenase activity (GO:0004497) were enriched (Fig. 3d; Additional file 8: Table S5).

\section{Kyoto encyclopedia of genes and genomes (KEGG)} pathway enrichment analysis

The DEGs were mainly enriched in 33 KEGG pathways, including cellular processes, environmental information processing, genetic information processing, metabolism, and organismal systems (Fig. 4a; Additional file 9: Table S6). The top 20 enriched KEGG pathways ( $Q$ value is arranged in ascending order) were used to draw a bubble chart (Fig. 4b; Additional file 9: Table S6). The results showed that the DEGs were enriched in phenylpropane biosynthesis (00940), biosynthesis of secondary metabolites (01110), metabolic pathways (01100), MAPK signaling pathways (04016), and plant hormone signal transduction (04075). Next, we performed gene set enrichment analysis (GSEA) using the genes related to plant signal transduction from the results of KEGG enrichment analysis (Fig. 4c; Additional file 10: Table S7) and obtained 109 genes positively related to the phenotype of AM seedlings, of which 9 genes were related to IAA (Fig. 4d). Interestingly, the GO enrichment results showed that all 9 genes were enriched in the nucleus (GO: 0005634), binding domain containing DNA (GO: 0003677), and were all enriched in the auxin activation signal pathway (GO: 0009734) and transcriptional regulation pathways (GO: 0006355). In addition, ARF5, an auxin regulator, was enriched in the biological process of hormone response (GO:0009725). Interestingly, the promoters of NADP-ME1 and NADP-ME2 contained the specific binding sites for ARF5 (Fig. 4e), indicating that ARF5 might directly regulate the expression of NADP-ME1 and NADP-ME2.

\section{Effects of AMF on endogenous root hormone}

Transcriptome analysis showed that genes related to plant hormone signal transduction responded to AMF colonization. Therefore, we analyzed the concentrations of IAA, gibberellin $\left(\mathrm{GA}_{3}\right)$, abscisic acid (ABA), cytokinin 


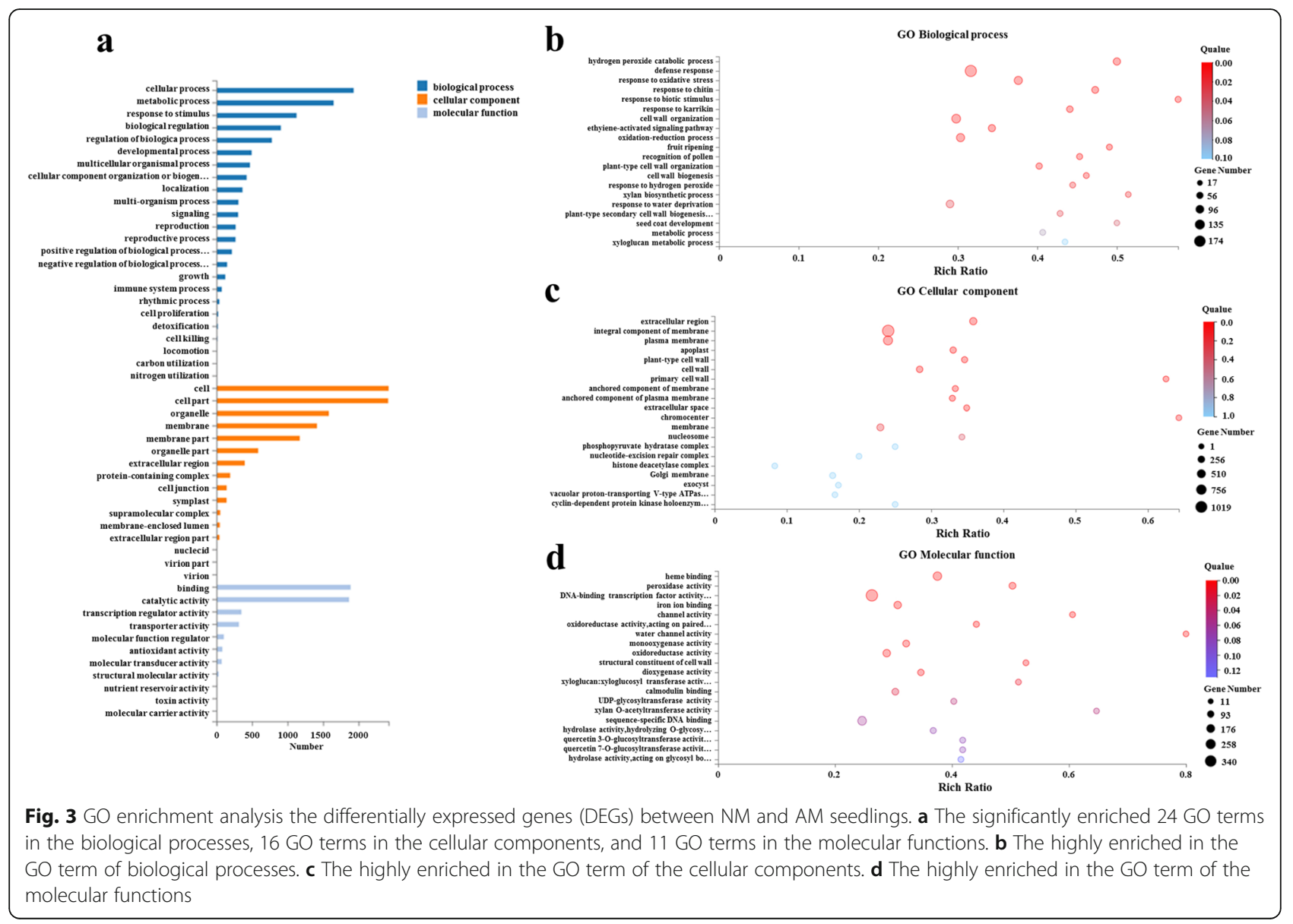

(CTK), and strigolactone (SL) in the roots of tomato seedlings. The results showed that the concentrations of IAA and CTK in the roots of AM seedlings significantly increased, while the concentrations of $\mathrm{ABA}$ significantly decreased, and the concentrations of $\mathrm{GA}_{3}$ and SL did not change compared with those in the NM seedlings (Fig. 5). These results indicated that the AMF colonization of the roots of tomato seedlings modulated endogenous hormone levels, which presumably further affected other physiological and metabolic processes.

\section{Response of NADP-MEs to hormones}

To verify whether hormones mediated root activity, tomato seedlings were treated with IAA, $\mathrm{GA}_{3}, \mathrm{ABA}, \mathrm{CTK}$, and SL, and qPCR was used to test the dynamic expression patterns of NADP-ME1 and NADP-ME2. The results showed that these 2 genes responded to all 5 hormones (Additional file 11: Figure S4). The expression of NADP-ME1 and NADP-ME2 was upregulated by IAA, and the gene expression remained highly upregulated from $1 \mathrm{~h}$ to $12 \mathrm{~h}$ after treatment, and the expression characteristics of these 2 genes were consistent (Additional file 11: Figure S4a). However, ABA significantly reduced the expression of $N A D P-M E 1$ and NADP-ME2 (Additional file 11: Figure S4c).

\section{Effects of IAA on AMF-induced root activity}

To clarify the role of IAA in AMF-induced root activity, we investigated the effects of IAA and 2,3,5-triiodobenzoic acid (TIBA, an auxin polar transport inhibitor) on root activity, NADP-ME activity, and NADP-ME1 and NADP-ME2 expression levels in tomato seedlings cultivated in continuous cropping substrate with or without AMF inoculation. The application of IAA significantly promoted tomato seedlings growth after $15 \mathrm{~d}$ of treatment, while the application of TIBA significantly inhibited the growth of tomato seedlings compared with that in the NM seedlings (Fig. 6a, b). Furthermore, the root activity of $\mathrm{AM}$ and tomato seedlings cultivated in the continuous cropping substrate irrigated with IAA (NI) increased by 65 and 27\%, respectively, while the root activity of tomato seedlings cultivated in the continuous cropping substrate irrigated with TIBA (NT) decreased by $54 \%$ compared with that in NM seedlings (Fig. 6c). Strikingly, the root activity of tomato seedlings cultivated in the continuous cropping substrate inoculation with AMF and irrigated with TIBA (AT) was significantly 

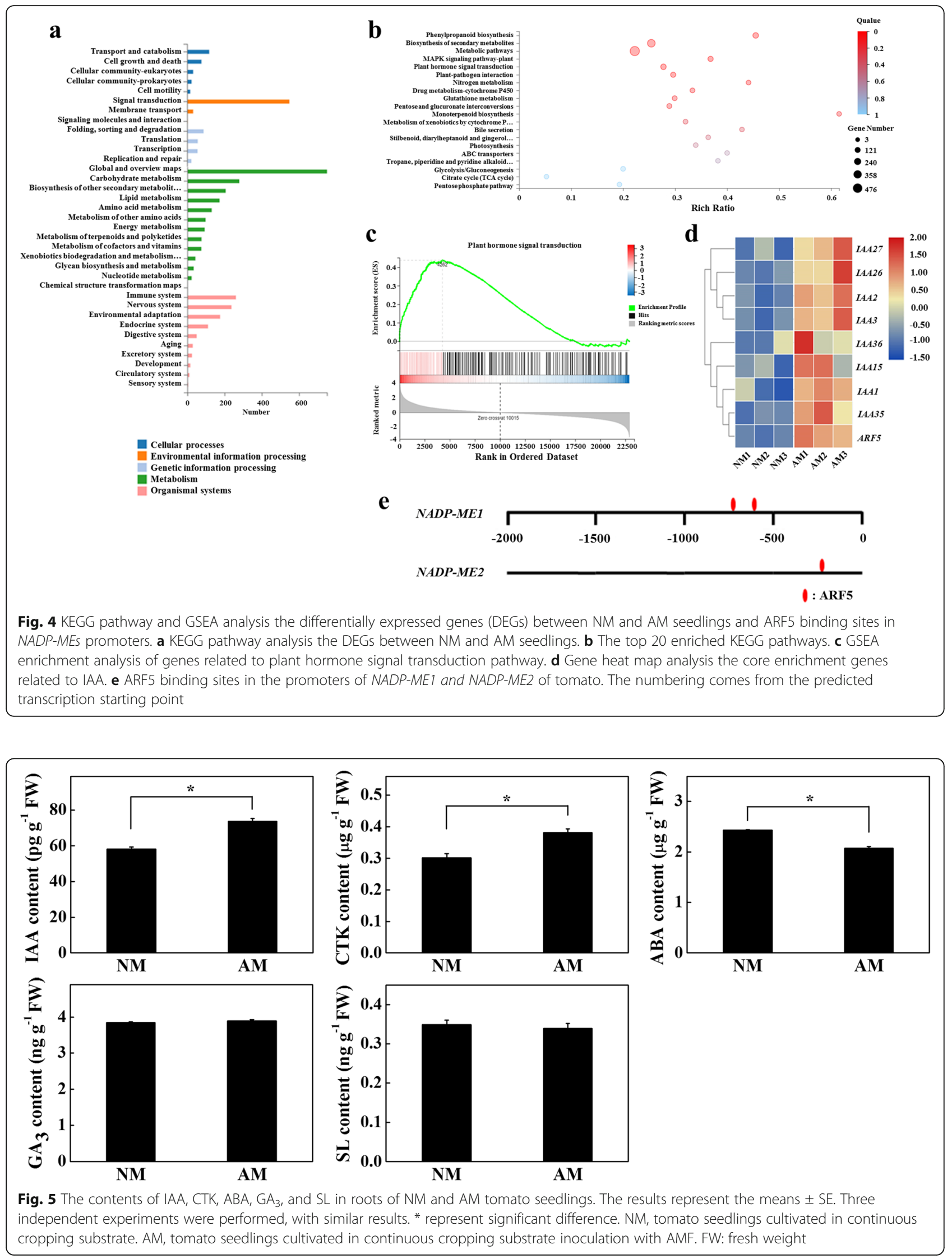
a

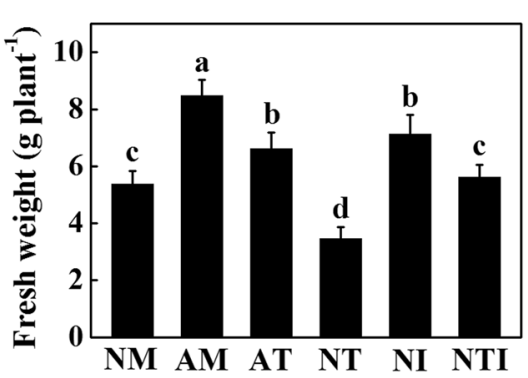

c

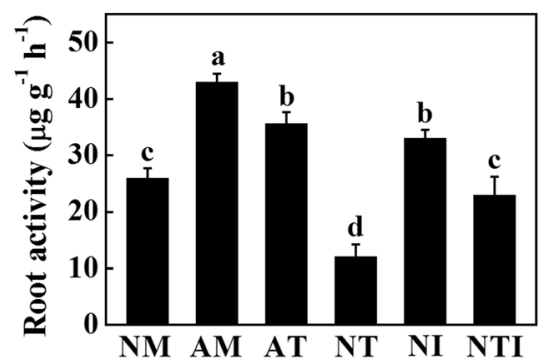

$\mathbf{e}$

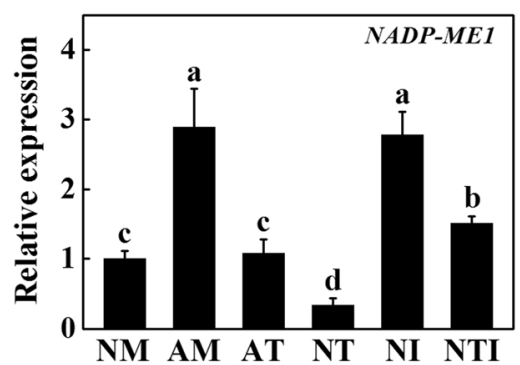

$\mathbf{b}$
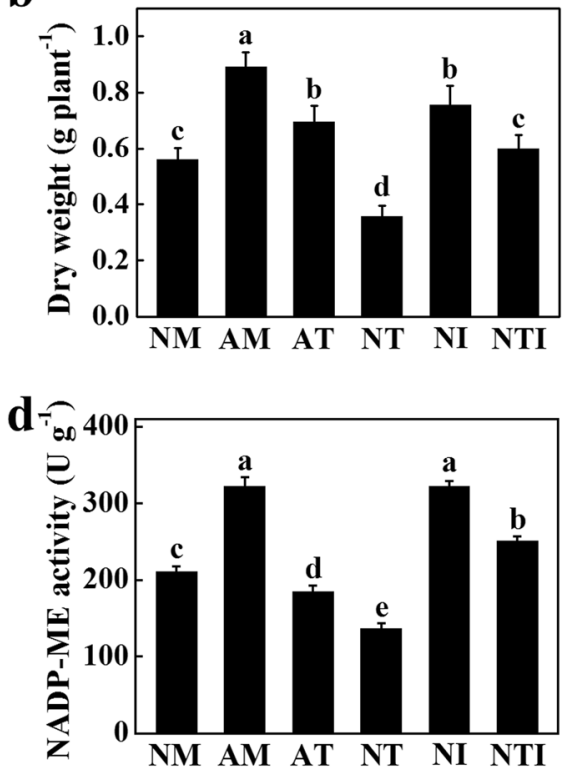

f

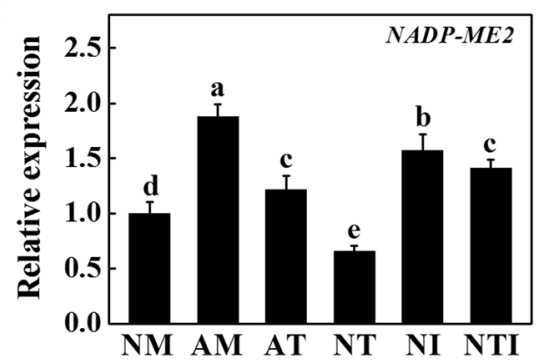

Fig. 6 IAA mediated AMF-induced growth, root and NADP-ME activity in tomato seedlings. a Fresh weight. b Dry weight. c The root activity of tomato seedlings. $\mathbf{d}$ The NADP-ME activity of tomato seedlings. e The expression of NADP-ME1. $\mathbf{f}$ The expression of NADP-ME2. After inoculation with AMF for $15 \mathrm{~d}$, IAA and TIBA were irrigated every $5 \mathrm{~d}$, and the data were determined at $30 \mathrm{~d}$. The results represent the means \pm SE. Means with the same letter did not significantly differ at $P<0.05$, according to Tukey's test. Three independent experiments were performed, with similar results. NM: tomato seedlings cultivated in the continuous cropping substrate; AM: tomato seedlings cultivated in the continuous cropping substrate inoculation with AMF; AT: tomato seedlings cultivated in the continuous cropping substrate inoculation with AMF and irrigated with TIBA; NT: tomato seedlings cultivated in the continuous cropping substrate irrigated with TIBA; NI: tomato seedlings cultivated in the continuous cropping substrate irrigated with IAA; NTI: tomato seedlings cultivated in the continuous cropping substrate irrigated with TIBA, and then irrigated with IAA after $24 \mathrm{~h}$

lower than that in the AM seedlings (Fig. 6c). As shown in Fig. 6d, the NADP-ME activity of AT seedlings markedly decreased compared with that in the AM seedlings. The NADP-ME activity of NI seedlings significantly increased compared with that in the NM seedlings (Fig. $6 \mathrm{~d})$. In contrast, the NADP-ME activity of NT seedlings decreased compared with that of NM seedlings (Fig. 6d). However, the inhibitory effects of TIBA were ameliorated by the application of IAA (Fig. 6d).

Gene expression results showed that the expression of NADP-ME1 and NADP-ME2 in AM plants was higher than that in NM plants (Fig. 6e, f). Compared with NM and AM, TIBA significantly reduced the expression of NADP-ME1 and NADP-ME2 in NT and AT plants; in contrast, the application of IAA significantly increased the expression of $N A D P-M E 1$ and $N A D P-M E 2$ (Fig. 6e, f). The gene expression of NADP-ME1 and NADP-ME2 in tomato seedlings cultivated in the continuous cropping substrate irrigated with TIBA, and then irrigated with IAA after $24 \mathrm{~h}$ (NTI) was consistent with the trend of NADP-ME enzyme activity (Fig. 6d-f). The above results indicated that IAA mediated the expression of $N A D P-M E 1$ and NADP-ME2 and root activity of tomato seedlings in continuous cropping substrates.

\section{Discussion}

AMF promoted tomato seedling growth in continuous cropping substrate

Compared with fresh substrates, continuous cropping substrates have many limitations, such as lower $\mathrm{pH}$ value, 
decreased nutrient content, reduced permeability, and imbalanced microbial community structure [3]. Recently, the use of beneficial microorganisms has become increasingly popular to maintain soil fertility and crop productivity [21]. AMF play a critical role in plant response to biotic and abiotic stresses [15, 22-24]. AMF can reduce the risk of ionic toxicity and cell membrane damage by decreasing $\mathrm{Na}^{+}$absorption and increasing antioxidant enzyme activity in cucumber [25]. Studies have shown that AMF significantly improve the physicochemical properties and enzyme activities of continuous cropping systems and promote the absorption of nutrients, resulting in promoted plant growth and increased yield $[15,16,26]$. Consistent with the previous studies, AMF incorporation into the continuous cropping substrate remarkably improved the growth of tomato seedlings and enhanced the yield of tomato (Fig. 1; Additional file 3: Figure S2). These results indicate that the inoculation of AMF in continuous cropping substrates is an effective way to relieve continuous cropping obstacles.

\section{AMF induced an increase in endogenous IAA in tomato roots}

It has been shown that the inoculation of AMF can regulate hormone levels in plants $[27,28]$. In this study, the inoculation of AMF significantly increased IAA and CTK concentrations in tomato roots and significantly reduced ABA concentrations (Fig. 5). Similarly, AMF increased the levels of IAA and CTK, decreased ABA levels, and enhanced hormone homeostasis in the damaged roots of maize [29]. Furthermore, AMF inoculation significantly increased the IAA content in the root system of trifoliate orange and decreased IAA loss in the root system [30]. In this study, the enrichment analysis of the KEGG pathway showed that the plant hormone signal transduction pathway was relatively enriched (Fig. 4b). Through GSEA, 109 genes were positively correlated with the AM phenotype, among which 9 genes were correlated with IAA (Fig. 4c, d). Similarly, AMF can increase the IAA level and promote the growth and development of host plants under stress by upregulating the expression of IAA transporter genes and downregulating the expression of auxin efflux genes [31]. In addition, AMF have a positive effect on the regulation of IAA levels in plants under drought [32], salt stress [33] and biotic stress [34]. These results suggested that the increase in IAA levels in tomato roots induced by AMF might be an important reason to promote the growth and development of tomato seedlings in continuous cropping substrates.

\section{IAA mediated AMF-induced NADP-MEs expression and NADP-ME activity}

NADP-ME is a cytoplasmic protein that is expressed during the development of tomato roots, stems, leaves, and fruits [35]. In particular, NADP-ME1 is involved in the regulation of malic acid content in the root apex, and the absence of NADP-ME1 results in an increase in malic acid content. Furthermore, NADP-ME1 also affects the signal transmission process [36]. However, NADP-ME2 plays an important role in the defense mechanism of plants and seems to be involved in the production of reactive oxygen species [37]. Our results showed that NADP-ME1 and NADP-ME2 responded to IAA, $\mathrm{GA}_{3}, \mathrm{ABA}, \mathrm{CTK}$, and SL (Additional file 11: Figure S4). Among them, IAA significantly increased the expression of NADP-ME1 and NADP-ME2 (Additional file 11: Figure S4a), which indicated that the induction of NADP-ME1 and NADP-ME2 by IAA might be a response to the stress of tomato under continuous cropping substrates. Notably, hormones play a crucial role in regulating complex signaling networks in different plant growth and development processes and in plant responses to environmental stresses [38]. Likewise, IAA is involved in regulating the defense response to various biotrophic and necrotrophic pathogens [38]. Our results showed that AMF inoculation significantly increased the IAA concentration in tomato roots (Fig. 5). Furthermore, IAA application significantly increased the expression levels of NADP-ME1 and NADP-ME2, NADP-ME activity, root activity, and the growth of tomato seedlings cultivated in continuous cropping substrates (Fig. 6). However, these effects were compromised when plants were treated with TIBA (Fig. 6). Similarly, AMF inoculation promotes the accumulation of endogenous IAA to improve the root system and nutrient absorption under stress conditions [39]. Exogenous IAA significantly increases the activity of NADP-ME in Malus baccata (L.) Borkh. [40]. Thus, AMF inoculation improved the endogenous IAA level in plant roots to increase the expression of NADP-ME1 and NADP$M E 2$ and NADP-ME activity.

\section{Conclusions}

In conclusion, our study revealed that IAA mediates the AMF-promoted tomato growth and NADP-MEs expression in continuous cropping substrates. Our study suggests that the use of AMF can effectively improve the growth of tomato cultivated in continuous cropping substrates, thereby showing a promising solution for the reuse of continuous cropping substrates with AMF.

\section{Methods}

\section{Plant materials and treatments}

Tomato (Solanum lycopersicum L. cv hezuo 903, obtained from Shanghai Changzhong Tomato Seed Industry Co., Ltd.) was used in this study. The AMF used in this study was an isolate of Funneliformis mosseae (BGC HEB07B, 1511C0001BGCAM0049, obtained from 
Huaian Chaimihe Agriculture Science and Technology Co., Ltd.), which was obtained through propagation with maize (Zea mays L.) as previously described [41].

The continuous cropping substrate, which had been used for the cultivation (complete growth cycle) of pepper and eggplant, was sterilized and then used to cultivate tomato in this study. The physical properties of the continuous cropping substrate deteriorated, the bulk density (BD) significantly increased, and the values of $\mathrm{pH}$ and electrical conductivity (EC) decreased (Additional file 12: Table S8).

The tomato seeds were placed on moist filter paper and germinated at $28^{\circ} \mathrm{C}$ for $30 \mathrm{~h}$ in the dark. After germination, the seeds were sown in plastic pots containing continuous cropping substrate or continuous cropping substrate inoculated with $F$. mosseae at a dose of 600 spores per plant. Seedlings were cultivated in an artificial growth chamber. The growth conditions were as follows: $25 \pm 2{ }^{\circ} \mathrm{C} / 18 \pm 2{ }^{\circ} \mathrm{C}$ (day/night), $12 \mathrm{~h} / 12 \mathrm{~h}$ (day/night) photoperiod, $60-75 \%$ relative humidity, and $300 \mu \mathrm{mol}$ $\mathrm{m}^{-2} \mathrm{~s}^{-1}$ light density. The growth parameters, root morphology, and Pn of tomato seedlings were determined at $40 \mathrm{~d}$. The enzyme activity related to root activity was measured at $20,25,30,35$, and $40 \mathrm{~d}$. The transcriptome sequencing was performed at $30 \mathrm{~d}$ with 3 biological replicates.

\section{Hormone treatments}

Tomato seedlings were cultured in fresh substrate and grown under in the same conditions as described above. The tomato seedlings were watered with $50 \mathrm{ml}$ of $100 \mu \mathrm{M} \mathrm{GA}_{3}$ (Solarbio, Beijing, China), IAA (Solarbio, Beijing, China), ABA (Solarbio, Beijing, China), CTK (Solarbio, Beijing, China), and SL (Solarbio, Beijing, China) solution per plant, respectively, and the control plants were watered with the same volume of deionized water. Root samples were taken at $0,1,6,12$, and $24 \mathrm{~h}$, respectively, to determine the gene expression of NADPMEs.

\section{IAA and TIBA treatment}

To clarify the relationship between IAA and tomato root activity and NADP-MEs, 15-d-old NM and AM tomato seedlings were watered with $50 \mathrm{ml}$ of $100 \mu \mathrm{M}$ IAA or TIBA (Aladdin, Shanghai, China), every $5 \mathrm{~d}$, and the control plants were watered with the same volume of deionized water. There were 6 treatments in the experiment, including NM, AM, AT, NT, NI, and NTI. Root samples were taken at $30 \mathrm{~d}$ to determine the root activity, enzyme activities, and NADP-MEs expression.

\section{Mycorrhizal colonization rate measurement}

For measurement of the rate of mycorrhizal colonization, fresh roots were collected at $30 \mathrm{~d}$, cleaned and cut to $1-2 \mathrm{~cm}$ and then incubated in $10 \%(\mathrm{w} / \mathrm{v})$ $\mathrm{KOH}$ (Sinopharm Chemical Reagent, Shanghai, China) at $90{ }^{\circ} \mathrm{C}$ for $40 \mathrm{~min}$. The roots were rinsed with distilled water and then soaked in $2 \%$ lactic acid (Solarbio, Beijing, China) at $90^{\circ} \mathrm{C}$ for $20 \mathrm{~min}$. Then, the roots were stained with $0.05 \%$ Trypan Blue dye (Solarbio, Beijing, China) at $90^{\circ} \mathrm{C}$ for $30 \mathrm{~min}$. After cooling, the root samples were decolored with destaining solution (distilled water: lactic acid: glycerin [Sinopharm Chemical Reagent, Shanghai, China] $=1: 2: 2, \mathrm{v} / \mathrm{v}$ ) for $2-4 \mathrm{~d}$ at room temperature. Thirty pieces of randomly selected stained root fragments were observed with a Leica DM1000 microscope (Leica Microsystems, Wetzlar, Hesse, Germany) to confirm the presence of fungal structures, including intraradical mycelia, vesicles, and arbuscules. The root colonization rate was measured as previously described [42].

\section{Morphological index and Pn measurement}

The plant height and stem diameter were measured at $40 \mathrm{~d}$ according to previously described methods [43, 44]. After removing the plants from the cultivation pot, the roots were washed with distilled water and the fresh weight was measured with an electronic balance (OHAUS, Parsippany, NJ, USA). The plant materials were enclosed in envelopes and placed in an oven (Shanghai Yiheng Scientific Instrument Co., Ltd., Shanghai, China) at $105^{\circ} \mathrm{C}$ for $30 \mathrm{~min}$. Then, the oven temperature was adjusted to $75^{\circ} \mathrm{C}$ for $2 \mathrm{~d}$ to obtain the dry weight. A WinRHIZO LA2400 root scanner system (Regent Instruments Inc., Québec, QC, Canada) was used to collect root morphological indexes.

The $\mathrm{Pn}$ was measured with a portable photosynthesis measurement system (Li-6400; Li-COR, Lincoln, NE, USA) after $1 \mathrm{~h}$ of light in the morning.

\section{Root activity and related enzyme activities}

The root activity of tomato was determined with the triphenyltetrazolium chloride (TTC) method as previously described [45].

The activities of 6PGDH, G6PDH, NADP-IDCH and NADP-ME were measured by spectrophotometry, recording the reduction of NADP at $340 \mathrm{~nm}[46,47]$. The experiment was conducted at $25^{\circ} \mathrm{C}$, and the reaction system volume was $1 \mathrm{ml}$, including $50 \mathrm{mM}$ HEPES (pH 7.6, Solarbio, Beijing, China), $2 \mathrm{mM} \mathrm{MgCl}_{2}$ (Sinopharm Chemical Reagent, Shanghai, China), $0.8 \mathrm{mM}$ NADP (Solarbio, Beijing, China) and plant samples. The reaction was initiated by adding $5 \mathrm{mM}$ 6-phosphate gluconate (Aladdin, Shanghai, China), $5 \mathrm{mM}$ glucose 6phosphate (Solarbio, Beijing, China), $10 \mathrm{mM}$ 2R,3S-isocitrate (Aladdin, Shanghai, China) and $10 \mathrm{mM}$ malic acid (Solarbio, Beijing, China), respectively. 


\section{Analysis of the contents of IAA, GA $3, C T K, A B A$, and $S L$ in tomato roots}

The hormone contents were determined at $30 \mathrm{~d}$ as previously described [48]. Tomato root tissue was fully ground in liquid nitrogen, and transferred to a precooled $50-\mathrm{ml}$ centrifuge tube that contained $4 \mathrm{ml}$ of precooled $80 \%$ chromatographic methanol (Aladdin, Shanghai, China). The mixture was placed on ice in the dark for $12 \mathrm{~h}$. The tubes were centrifuged at $10000 \mathrm{~g}$ and $4{ }^{\circ} \mathrm{C}$ for $15 \mathrm{~min}$, and the supernatant was transferred to another 50 - $\mathrm{ml}$ tube and stored in a refrigerator at $4{ }^{\circ} \mathrm{C}$. Afterward, $3 \mathrm{ml}$ of $80 \%$ methanol was added to the remaining precipitate 3 times, and the supernatants were combined. Then, $1.0 \mathrm{~g}$ of polyvinylpyrrolidone (Sinopharm Chemical Reagent, Shanghai, China) was added to the supernatant and shaken in a shaker at $4{ }^{\circ} \mathrm{C}$ in the dark for $1 \mathrm{~h}$. Subsequently, the mixture was centrifuged at $10000 \mathrm{~g}$ and $4{ }^{\circ} \mathrm{C}$ for $15 \mathrm{~min}$, and the supernatant was passed through a C18 extraction cartridge (Waters, Milford, MA, USA) that had already been rinsed in the dark. The liquid was stored in a 50-ml centrifuge tube and freeze-dried in vacuum for $3 \mathrm{~d}$ under dark conditions. Then, $1 \mathrm{ml}$ of precooled chromatographic methanol was added to the tube to completely dissolve the hormone, and the samples were filtered with a $0.45-\mu \mathrm{m}$ organic microfiltration membrane before loading. The samples were detected with a high-performance liquid chromatography 1525 system (Waters, Milford, MA, USA).

\section{qPCR analysis}

Total RNA was isolated from tomato roots with the RNA Simple Total RNA Kit (Tiangen, Beijing, China). Total RNA $(1 \mu \mathrm{g})$ was reverse transcribed into cDNA using HiScript $^{\circ}$ II Q RT SuperMix (+ gDNA-wiper) (Vazyme, Nanjing, China) for qPCR. qPCR assays were performed using ChamQ Universal SYBR-qPCR Master Mix (Vazyme, Nanjing, China) in a StepOne (TM) real-time PCR system (Applied Biosystems, Foster, CA, USA). The tomato Ubi3 gene was used as an internal control. The primer sequences are shown in Additional file 13: Table S9. The relative gene expression was calculated as described by Livak and Schmittgen [49].

\section{RNA extraction, cDNA library construction and Illumina sequencing}

Total RNA was extracted from the roots of tomato seedlings using TRIzol Reagent (Invitrogen, Carlsbad, CA, USA). The RNA quality and purity were verified by a Nanodrop 2000 (Thermo Fisher Scientific, Rockford, IL, USA) and electrophoresis in a 1.0\% agarose gel. The mRNAs were purified from total RNA using poly-T oligo-attached magnetic beads (Invitrogen, Carlsbad, CA, USA). Subsequently, the mRNAs were fragmented, and cDNA was synthesized using random hexamers, DNA polymerase I (Thermo Fisher Scientific, Rockford, IL, USA) and RNase H (Thermo Fisher Scientific, Rockford, IL, USA). The purified double-stranded cDNAs were ligated to adaptors for Illumina paired-end sequencing. An Agilent 2100 Bioanalyzer (Agilent Technologies, Santa Clara, CA, USA) and ABI real-time RT-PCR (Applied Biosystems, Foster, CA, USA) were used to verify the quality and quantity of the library, respectively. The cDNA libraries were sequenced with the Illumina HiSeq2000 platform (Illumina, San Diego, CA, USA) by the Beijing Genomics Institute.

\section{Sequence data analysis and annotation}

After the raw reads, adaptor sequences and low-quality reads were removed, all the clean reads were mapped to the tomato reference genome using TopHat v1.4.0 [50]. The transcript abundance was normalized by the fragments per kilobase of exon per million fragments mapped using Cufflinks [51].

\section{Identification of DEGs}

The significance of the gene expression difference was recognized based on the false discovery rate (FDR) value less than 0.01 and $\mid \log _{2}$ (fold change) $\mid \geq 2$. After normalization, hierarchical clustering and k-means clustering analysis of the expression patterns were performed using Mutiexperimental Viewer v4.7 [52].

\section{GO and KEGG enrichment analysis}

For identification of putative biological functions and pathways of the DEGs, the GO and KEGG database were searched for annotation. GO classification was performed by WEGO [53]. The AgriGO and KOBAS2.0 packages were used to analyze the enrichment of GO and KEGG at a significance cutoff of 0.05 FDR, respectively $[54,55]$.

\section{Statistical analysis}

The experiment was carried out in a completely randomized design with three independent replicates, and each replicate contained 12 plants. Significant differences $(P<0.05)$ between treatments were determined using Tukey's test.

\section{Supplementary Information}

The online version contains supplementary material available at https://doi. org/10.1186/s12870-020-02817-2.

Additional file 1: Figure S1. The colonization of AMF in tomato seedlings.

Additional file 2: Table S1. Effects of AMF inoculation on tomato root growth. 
Additional file 3: Figure S2. Effects of arbuscular mycorrhizal fungi (AMF) inoculation on the yield of tomato.

Additional file 4: Table S2. Base statistics after filtering from NM1, NM2, NM3, AM1, AM2 and AM3 libraries.

Additional file 5: Table S3. Mapping results of clean reads against the tomato genome and gene.

Additional file 6: Figure S3. Gene heat map showing the gene expression differences between NM and AM seedling roots.

Additional file 7: Table S4. The differentially expressed genes between $\mathrm{NM}$ and $\mathrm{AM}$

Additional file 8: Table S5. GO enrichment analysis the differentially expressed genes between NM and AM.

Additional file 9: Table S6. KEGG pathway analysis the differentially expressed genes between NM and AM.

Additional file 10: Table S7. GSEA enrichment analysis of genes related to plant hormone signal transduction pathway.

Additional file 11: Figure S4. Responses of NADP-ME1 and NADP-ME2 to hormones.

Additional file 12: Table S8. Comparison of physical and chemical properties of continuous cropping substrate and fresh substrate.

Additional file 13: Table S9. Primers used for $q P C R$ assays.

\section{Abbreviations}

6PGDH: 6-phosphate gluconate dehydrogenase; ABA: Abscisic acid; AM: Tomato seedlings cultivated in the continuous cropping substrate inoculation with AMF; AMF: Arbuscular mycorrhizal fungi; AT: Tomato seedlings cultivated in the continuous cropping substrate inoculation with AMF and irrigated with TIBA; CTK: Cytokinin; DEGs: Differentially expressed genes; EC: Electrical conductivity; FDR: False discovery rate; FW: Fresh weight; G6PDH: Glucose-6-phosphate dehydrogenase; GA : Gibberellin; GO: Gene ontology; GSEA: Gene set enrichment analysis; IAA: Indole acetic acid; KEGG: Kyoto encyclopedia of genes and genomes; NADP-IDCH: NADPisocitrate dehydrogenase; NADP-ME: NADP-malic enzyme; NI: Tomato seedlings cultivated in the continuous cropping substrate irrigated with IAA; NM: Tomato seedlings cultivated in the continuous cropping substrate; NT: Tomato seedlings cultivated in the continuous cropping substrate irrigated with TIBA; NTI: Tomato seedlings cultivated in the continuous cropping substrate irrigated with TIBA, and then irrigated with IAA after 24 h; Pn: Net photosynthetic rate; SL: Strigolactone; TIBA: 2,3,5-triiodobenzoic acid

\section{Acknowledgements}

Not applicable.

\section{Authors' contributions}

J.S. and S.S. designed the experiment. Y.W. and W.Z.Z. performed the experiments and wrote the manuscript; W.K.L. and W.W.X. collected plant materials and prepared figures; G.J.A., S.S. and S.R.G. analyzed the data and modified this manuscript. All authors reviewed and approved the manuscript.

\section{Funding}

This work was supported by the National Key Research and Development Program of China (2019YFD1001901, 2019YFD1001902), the National Natural Science Foundation of China $(31872152,31801902)$ and the China Postdoctoral Science Foundation (2019 T120433). The supporters did not play any role in the design, collection, analysis, interpretation of the relevant data, or in writing the manuscript.

\section{Availability of data and materials}

The data charts supporting the results and conclusions are included in the article and additional files. All sequences generated by sequencing for this study are available in the NCBI Sequence Read Archive (SRA) database under Bioproject PRJNA686719 (http://www.ncbi.nlm.nih.gov/bioproject/686719). The materials are available upon request by contacting the corresponding author.
Ethics approval and consent to participate

Not applicable.

\section{Consent for publication}

Not applicable.

\section{Competing interests}

The authors declare that they have no competing interests.

\section{Author details}

${ }^{1}$ College of Horticulture, Nanjing Agricultural University, Nanjing 210095 , China. ${ }^{2}$ College of Horticulture and Plant Protection, Henan University of Science and Technology, Luoyang 471023, China.

Received: 9 September 2020 Accepted: 22 December 2020

Published online: 18 January 2021

\section{References}

1. Tzortzakis N, Nicola S, Savvas D, Voogt W. Editorial: soilless cultivation through an intensive crop production scheme. management strategies, challenges and future directions. Front Plant Sci. 2020;11:363.

2. Sun J, Gao HB, Tian J, Wang JW, Du CX, Guo SR. Development status and trends of protected horticulture in China. J Nanjing Agr Uni. 2019;42:594604 (in Chinese).

3. Jin L, Lu J, Yu JH, Jin N, Xie JM, Xiao XM. Mitigation effect of exogenous silicon on inhibiting cucumber seed germination by continuous cropping matrix extract. Acta Bot Boreal Occident Sin. 2019;39:1600-8 (in Chinese).

4. Li W, Cheng ZH, Meng HW, Zhou J, Liang J, Liu XJ. Effect of rotating different vegetables on micro-biomass and enzyme in tomato continuous cropped substrate and afterculture tomato under plastic tunnel cultivation. Acta Hortic Sin. 2012;39(1):73-80 (in Chinese).

5. Zou CJ, Zhang YY, Zhang YM, Guo X, Li MJ, Li TL. Regulation of biochar on matrix enzyme activities and microorganisms around cucumber roots under continuous cropping. Chin J Appl Ecol. 2015;26(6):1772-8 (in Chinese).

6. Lyu J, Jin L, Jin N, Xie JM, Xiao XM, Hu LL, Tang ZQ, Wu Y, Niu LJ, Yu JH. Effects of different vegetable rotations on fungal community structure in continuous tomato cropping matrix in greenhouse. Front Microbiol. 2020; 11:829.

7. Jie W, Lin J, Guo N, Cai B, Yan X. Community composition of rhizosphere fungi as affected by Funneliformis mosseae in soybean continuous cropping soil during seedling period. Chilean J Agric Res. 2019;79(3):356-65.

8. Smith SE, Smith FA, Jakobsen I. Mycorrhizal fungi can dominate phosphate supply to plants irrespective of growth responses. Plant Physiol. 2003;133(1): $16-20$.

9. Wright SF, Upadhyaya A. A survey of soils for aggregate stability and glomalin, a glycoprotein produced by hyphae of arbuscular mycorrhizal fungi. Plant Soil. 1998;198(1):97-107.

10. Zhou J, Zang H, Loeppmann S, Gube M, Kuzyakov Y, Pausch J. Arbuscular mycorrhiza enhances rhizodeposition and reduces the rhizosphere priming effect on the decomposition of soil organic matter. Soil Biology Biochem. 2020;140:107641.

11. Dimkpa C, Weinand T, Asch F. Plant-rhizobacteria interactions alleviate abiotic stress conditions. Plant Cell Environ. 2009:32(12):1682-94.

12. Mathur S, Tomar RS, Jajoo A. Arbuscular Mycorrhizal fungi (AMF) protects photosynthetic apparatus of wheat under drought stress. Photosynth Res. 2019;139:227-38.

13. Gu S, Wu S, Guan Y, Zhai C, Zhang Z, Bello A, Guo X, Yang W. Arbuscular mycorrhizal fungal community was affected by tillage practices rather than residue management in black soil of Northeast China. Soil Tillage Res. 2020; 198:104552.

14. Kong L, Gong X, Zhang X, Zhang W, Sun J, Chen B. Effects of arbuscular mycorrhizal fungi on photosynthesis, ion balance of tomato plants under saline-alkali soil condition. J Plant Nutr. 2020;43(5):682-98.

15. Chen K, Liu W, Guo S, Liu R, Li M. Diversity of arbuscular mycorrhizal fungi in continuous cropping soils used for pepper production. Afr J Microbiol Res. 2012:6(10):2469-74.

16. Jie W, Liu X, Cai B. Diversity of rhizosphere soil arbuscular mycorrhizal fungi in various soybean cultivars under different continuous cropping regimes. PLoS One. 2013;8(8):e72898.

17. Li Y, Chen YL, Li M, Lin XG, Liu RJ. Effects of arbuscular mycorrhizal fungi communities on soil quality and the growth of cucumber seedlings in a 
greenhouse soil of continuously planting cucumber. Pedosphere. 2012;22(1): 79-87.

18. Deliopoulos T, Devine KJ, Haydock PPJ, Jones PW. Studies on the effect of mycorrhization of potato roots on the hatching activity of potato root leachate towards the potato cyst nematodes, Globodera pallida and G. rostochiensis. Nematology. 2007;9:719-29.

19. Chen S, Zhao H, Zou C, Li Y, Chen Y, Wang Z, Jiang Y, Liu A, Zhao P, Wang $M$, Ahammed GJ. Combined inoculation with multiple arbuscular mycorrhizal fungi improves growth, nutrient uptake and photosynthesis in cucumber seedlings. Front Microbiol. 2017;8:2516.

20. Zhou Y, Yang XW, Zhou SM, Wang YJ, Yang R, Xu FD, Mei JJ, Shen GY, Li QJ, He DX. Activities of key enzymes in root NADP-dehydrogenase system and their relationships with root vigor and grain yield formation in wheat. Sci Agr Sin. 2018;51(11):2060-71 (in Chinese).

21. Chen W, Teng Y, Li Z, Liu W, Ren W, Luo Y, Christie P. Mechanisms by which organic fertilizer and effective microbes mitigate peanut continuous cropping yield constraints in a red soil of South China. Appl Soil Ecol. 2018; 128:23-34.

22. Garg N, Chandel S. Arbuscular mycorrhizal networks: process and functions. Agron Sustain Dev. 2010;30(3):581-99.

23. Chen S, Jin W, Liu A, Zhang S, Liu D, Wang F, Lin X, He C. Arbuscular mycorrhizal fungi (AMF) increase growth and secondary metabolism in cucumber subjected to low temperature stress. Sci Hortic. 2013;160:222-9.

24. Ahammed GJ, Mao Q, Yan Y, Wu M, Wang Y, Ren J, Guo P, Liu A, Chen S. Role of melatonin in arbuscular mycorrhizal fungi-induced resistance to fusarium wilt in cucumber. Phytopathology. 2020;110(5):999-1009.

25. Ahmad H, Hayat S, Ali M, Liu T, Cheng Z. The combination of arbuscular mycorrhizal fungi inoculation (Glomus versiforme) and 28-homobrassinolide spraying intervals improves growth by enhancing photosynthesis, nutrient absorption, and antioxidant system in cucumber (Cucumis sativus L.) under salinity. Ecol Evol. 2018;8(11):5724-40.

26. Amaya-Carpio L, Davies FT, Fox T, He C. Arbuscular mycorrhizal fungi and organic fertilizer influence photosynthesis, root phosphatase activity, nutrition, and growth of Ipomoea carnea ssp fistulosa. Photosynthetica. 2009;47(1):1-10.

27. Bi Y, Xiao L, Sun J. An arbuscular mycorrhizal fungus ameliorates plant growth and hormones after moderate root damage due to simulated coal mining subsidence: a microcosm study. Environ Sci Pollut Res. 2019;26(11):11053-61.

28. Zhou YH, Ge SB, Jin L, Yao K, Wang Y, Wu X, Zhou J, Xia XJ, Shi K, Foyer CH, $\mathrm{Yu}$ JQ. A novel $\mathrm{CO}_{2}$-responsive systemic signaling pathway controlling plant mycorrhizal symbiosis. New Phytol. 2019;224(1):106-16.

29. Bi Y, Zhang J, Song Z, Wang Z, Qiu L, Hu J, Gong Y. Arbuscular mycorrhizal fungi alleviate root damage stress induced by simulated coal mining subsidence ground fissures. Sci Total Environ. 2019;652:398-405.

30. Zhang F, Wang P, Zou YN, Wu QS, Kuca K. Effects of mycorrhizal fungi on root-hair growth and hormone levels of taproot and lateral roots in trifoliate orange under drought stress. Arch Agron Soil Sci. 2019;65(9):1316-30.

31. Liu CY, Wang P, Zhang DJ, Zou YN, Kuca K, WU QS. Mycorrhiza-induced change in root hair growth is associated with IAA accumulation and expression of EXPs in trifoliate orange under two P levels. Sci Hortic. 2018; 234:227-35.

32. Marulanda A, Barea JM, Azcon R. Stimulation of plant growth and drought tolerance by native microorganisms (AM fungi and bacteria) from dry environments: mechanisms related to bacterial effectiveness. J Plant Growth Regul. 2009;28(2):115-24

33. Liu HG, Wang YJ, Hart M, Chen H, Tang M. Arbuscular mycorrhizal symbiosis regulates hormone and osmotic equilibrium of Lycium barbarum L. under salt stress. Mycosphere. 2016;7(6):828-43.

34. He L, Li C, Liu R. Indirect interactions between arbuscular mycorrhizal fungi and Spodoptera exigua alter photosynthesis and plant endogenous hormones. Mycorrhiza. 2017;27(6):525-35.

35. Knee M, Finger FL. NADP ${ }^{+}$-malic enzyme and organic-acid levels in developing tomato fruits. J Amer Soc Hort Sci. 1992;117(5):799-801.

36. Badia MB, Maurino VG, Pavlovic T, Arias CL, Pagani MA, Andreo CS, Saigo M, Drincovich MF, Gerrard Wheeler MC. Loss of function of Arabidopsis NADPmalic enzyme 1 results in enhanced tolerance to aluminum stress. Plant J. 2020;101(3):653-65.

37. Voll LM, Zell MB, Engelsdorf T, Saur A, Gerrard Wheeler M, Drincovich MF, Weber APM, Maurino VG. Loss of cytosolic NADP-malic enzyme 2 in Arabidopsis thaliana is associated with enhanced susceptibility to Colletotrichum higginsianum. New Phytol. 2012;195(1):189-202.
38. Bari $\mathrm{R}$, Jones JDG. Role of plant hormones in plant defence responses. Plant Mol Biol. 2009;69(4):473-88.

39. Hashem A, Abd allah EF, Alqarawi AA, Wirth S, Egamberdieva D. Comparing symbiotic performance and physiological responses of two soybean cultivars to arbuscular mycorrhizal fungi under salt stress. Saudi J Biol Sci. 2019;26(1):38-48.

40. Lang D, Lyu D, Zhu Z, Qin S. Exogenous glucose mediates the regulation of root morphology and carbon-nitrogen metabolism by indole-3-acetic acid (IAA) in Malus baccata (L.) Borkh. in soil with low organic carbon content. J Plant Growth Regul. 2019;38(4):1598-615.

41. Chaiyasen A, Chaiya L, Douds DD, Lumyong S. Influence of host plants and soil diluents on arbuscular mycorrhizal fungus propagation for on-farm inoculum production using leaf litter compost and agrowastes. Biol Agric Hortic. 2017;33(1):52-62.

42. Phillips JM, Hayman DS. Improved procedures for clearing roots and staining parasitic and vesicular-arbuscular mycorrhizal fungi for rapid assessment of infection. Trans Br Mycol Soc. 1970;55:158-61.

43. Tang YY, Yuan YH, Shu S, Guo SR. Regulatory mechanism of $\mathrm{NaCl}$ stress on photosynthesis and antioxidant capacity mediated by transglutaminase in cucumber (Cucumis sativus L.) seedlings. Sci Hortic. 2018;235:294-306.

44. Wang Y, Guo S, Wang L, Wang L, He X, Shu S, Sun J, Lu N. Identification of microRNAs associated with the exogenous spermidine-mediated improvement of high-temperature tolerance in cucumber seedlings (Cucumis sativus L.). BMC Genom. 2018;19:285.

45. Parida AK, Das AB, Mohanty P. Defense potentials to $\mathrm{NaCl}$ in a mangrove, Bruquiera parviflora: differential changes of isoforms of some antioxidative enzymes. J Plant Physiol. 2004;161(5):531-42.

46. Corpas FJ, Barroso JB, Sandalio LM, Palma JM, Lupianez JA, del Rio LA. Peroxisomal NADP-dependent isocitrate dehydrogenase. Characterization and activity regulation during natural senescence. Plant Physiol. 1999;121(3): 921-8.

47. Valderrama R, Corpas FJ, Carreras A, Gomez-Rodriguez MV, Chaki M, Pedrajas JR, Fernandez-Ocana A, Del Rio LA, Barroso JB. The dehydrogenasemediated recycling of $\mathrm{NADPH}$ is a key antioxidant system against saltinduced oxidative stress in olive plants. Plant Cell Environ. 2006;29(7):144959.

48. Wang Y, Gong XW, Liu WK, Kong L, Si XY, Guo SR, Sun J. Gibberellin mediates spermidine-induced salt tolerance and the expression of GT-3b in cucumber. Plant Physiol Biochem. 2020;152:147-56.

49. Livak KJ, Schmittgen TD. Analysis of relative gene expression data using real-time quantitative PCR and the $2^{-\Delta \Delta C T}$ method. Methods. 2001;25(4):402-

50. Trapnell C, Williams BA, Pertea G, Mortazavi A, Kwan G, van Baren MJ, Salzberg SL, Wold BJ, Pachter L. Transcript assembly and quantification by RNA-Seq reveals unannotated transcripts and isoform switching during cell differentiation. Nat Biotechnol. 2010:28(5):511-5.

51. Trapnell C, Roberts A, Goff L, Pertea G, Kim D, Kelley DR, Pimentel H, Salzberg SL, Rinn JL, Pachter L. Differential gene and transcript expression analysis of RNA-seq experiments with TopHat and cufflinks. Nat Protoc. 2012;7(3):562-78.

52. Saeed Al, Sharov V, White J, Li J, Liang W, Bhagabati N, Braisted J, Klapa M, Currier T, Thiagarajan M, Sturn A, Snuffin M, Rezantsev A, Popov D, Ryltsov A, Kostukovich E, Borisovsky I, Liu Z, Vinsavich A, Trush V, Quackenbush J. TM4: a free, open-source system for microarray data management and analysis. Biotechniques. 2003;34(2):374-8.

53. Ashburner M, Ball CA, Blake JA, Botstein D, Butler H, Cherry JM, Davis AP, Dolinski K, Dwight SS, Eppig JT, Harris MA, Hill DP, Issel-Tarver L, Kasarskis A, Lewis S, Matese JC, Richardson JE, Ringwald M, Rubin GM, Sherlock G. Gene ontology: tool for the unification of biology. Nat Genet. 2000;25(1):25-9.

54. Du Z, Zhou X, Ling Y, Zhang Z, Su Z. AgriGO: a GO analysis toolkit for the agricultural community. Nucleic Acids Res. 2010;38:64-70.

55. Xie C, Mao X, Huang J, Ding Y, Wu J, Dong S, Kong L, Gao G, Li CY, Wei L. KOBAS 2.0: a web server for annotation and identification of enriched pathways and diseases. Nucleic Acids Res. 2011;39:316-22.

\section{Publisher's Note}

Springer Nature remains neutral with regard to jurisdictional claims in published maps and institutional affiliations. 